\title{
Selection of Oleaginous Yeasts with Lipid Accumulation by the Measurement of Sudan Black B for Benefits of Biodiesel
}

\author{
Kusumawadee Thancharoen, Auttaporn Malasri, Walailak Leamsingkorn, and Patchareeporn Boonyalit \\ Department of Biology, Rajabhat MahaSarakham University, MahaSarakham, Thailand \\ Email: Kthancharoen@gmail.com, Pangpoond@hotmail.com
}

\begin{abstract}
Microbial lipids possess significant potential which can be explored for biodiesel production. This study attempted to screened oleaginous yeasts and selected the strain that accumulated the largest quantity of lipid for lipid production from glucose. Among the 24 yeast strains isolated from canteen wastewater, 10 oleaginous yeasts were selected by Sudan Black B test. Sudan Black B staining revealed the presence of lipid inclusion bodies in the cells during cultivation under nitrogen-limiting conditions. Total oleaginous strains that accumulated quantities of lipid higher than $20 \%$ of their biomass when cultivated in glucose. It was found that accumulated the CTWY07 highest lipid content, up to $71.59 \%$ of its biomass $(4.904 \mathrm{~g} / \mathrm{L}$ lipid and $6.85 \mathrm{~g} / \mathrm{L}$ biomass), while CTWY17 grew the fastest by shaking flask cultivation in glucose. The results show that the isolated yeasts could be promising candidates for biodiesel production.
\end{abstract}

Index Terms-biodiesel, lipid accumulation, oleaginous, screening, single cell oil, Sudan Black B

\section{INTRODUCTION}

Concern for the increase in energy demand and the depletion of fossil fuel reserves has resulted in a rapid rise in crude oil prices, and therefore, securing alternative sources of energy is urgently required. One of the most promising renewable energy resources is biodiesel, which is produced from renewable biomasses by transesterification of triacylglyceols, yielding monoalkyl esters of long-chain fatty acids with short-chain alcohols, for example, fatty acid methyl esters and fatty acid ethyl esters [1]. Biodiesel, with the properties of low pollution, high fuel value which is safer than fossil diesel, is becoming the most promising alternative energy source for crude oil. And the research of biodiesel is also becoming a key direction in energy research [2]. Biodiesel is usually produced from oleaginous crops, such as rapeseed, soybean, sunflower and palm, through a chemical transesterification process of their oils with short chain alcohols, mainly methanol. Besides addressing environmental concerns related to greenhouse gasses, biodiesel offers new income to farmers. However, traditional oil-rich crops are limited by land availability, as well as environmental and social issues regarding the

Manuscript received October 10, 2016; revised April 20, 2017. use of feed and food crops for fuel. In comparison to other vegetable oils and animal fats, the production of microbial oil has many advantages: microbes have a short life cycle as compared to plants so the time to harvest is shorter, there is less labor required, microbial oil production is less affected by venue, season and climate, and scale-up is easier. Oleaginous microorganisms are defined as oleaginous species with oil contents excess of $20 \%$ of biomass weight. Microbial oils, also called Single Cell Oils (SCO), are produced by some oleaginous microorganisms, such yeast, fungi, bacteria and microalgae. While the eukaryotic yeast, fungi and microalgae can synthetize triglycerol (TAG), which are similar with the composition of vegetable oils, prokaryotic bacteria can synthetize specific lipids [3]. Yeasts can grow on a variety of substrates, even inexpensive materials such as wastes of agriculture and industry, and thus oleaginous yeast strains that can efficiently produce lipid from low-cost raw materials are of great interest. Biolipids, including triacylglycerol produced by oleaginous yeast, have been confirmed to be one of the most important raw materials for biodiesel production. The quality of biodiesel depens upon the fatty acid composition of the biolipids [4]. In general, biolipids produced by oleaginous yeast are suitable feedstock for biodiesel, because the fatty acid composition satisfies important criteria i.e., chain length and saturation degree. However, the fatty acid composition of biolipids is strain specific, it is therefore important to select oleaginous yeast strains to ascertain their suitability for biodiesel production [5]. Among the oleaginous yeasts, Yarrowia, Candida, Rhodotorula, Rhodosporidium, Cryptococcus, Trichosporon and Lipomyces have been studied for their microbial oil properties. The oil accumulated by the yeasts are in the form of triacylglycerols that are predominantly oleic (18:1), linoleic (18:2), stearic (18:0), palmitic (16:0) or palmitoleic (16:1) [6]. In this study an attempt has been made to screening of lipid accumulated yeast strains from glucose as a carbon sources.

\section{MATERIALS AND METHODS}

\section{A. Yeast Strains}

Total 26 yeast strains were isolated from canteen wastewater (CTWY) in Rajabhat Maha Sarakham 
University, Maha Sarakham Province, Thailand, in JuneAugust 2015. The strains were stored on Malt extractGlucose-Yeast extract-Peptone (MGYP) agar slants (containing g/L : glucose, 10; peptone, 5; yeast extract, 5; malt extract, $3 ; \mathrm{KH}_{2} \mathrm{PO}_{4}, 1$ and $\left.\mathrm{MgSO}_{4} .7 \mathrm{H}_{2} \mathrm{O}, 0.5\right)$ at $4{ }^{\circ} \mathrm{C}$ for futher study.

\section{B. Sudan Black B Staining for Oleaginous Yeasts}

Yeast culture grown till early stationary phase from the slant were stained using original Sudan Black B staining protocol and the modified protocol [7]. Heat fix the smeared on a microscopic slide and air dry, flood the smear with Sudan Black B stain, keep for 15 minutes till the stain turns greenish blue. Wash the slide remove the stain and counter stain with $0.5 \%$ saffranin for 30 seconds, wash, dry and observe under microscope.

\section{Extraction of Lipids and Biomass from Isolated Strains}

The yeast strains which showed positive response with Sudan Black B staining, were cultivated in Erlenmeyer flasks, containing $30 \mathrm{ml}$ of inoculation media (MGYP) and incubated in an incubator shaker, at $150 \mathrm{rpm}$ and $28 \pm 2{ }^{\circ} \mathrm{C}$ for 24 hours. After 24 hours, initial cell concentration determined by optical density (OD) at 600 $\mathrm{nm}, 5 \mathrm{ml}$ of culture was transferred to $45 \mathrm{ml}$ of nitrogen limited fermentation media (containing (in $\mathrm{g} / \mathrm{l}$ ): glucose, 40; $\left(\mathrm{NH}_{4}\right)_{2} \mathrm{SO}_{4}, 2 ; \mathrm{KH}_{2} \mathrm{PO}_{4}, 7 ; \mathrm{NaH}_{2} \mathrm{PO}_{4}, 2 ; \mathrm{MgSO}_{4} .7 \mathrm{H}_{2} \mathrm{O}$ 1.5 and Yeast extract, 1) in $250 \mathrm{ml}$ Erlenmeyer flask on a rotary shaker (Lab Companion IS-971R, Korea) at 150 rpm and incubated at $28 \pm 2{ }^{\circ} \mathrm{C}$ for 120 hours. All media were sterilized by autoclaving for $15 \mathrm{~min}$ at $121^{\circ} \mathrm{C} .50 \mathrm{ml}$ of this culture was harvested by centrifugation at $5,000 \mathrm{~g}$ for 5 minutes, and dried at $60^{\circ} \mathrm{C}$ to constant mass. The biomass harvested biomass was washed twice with water was then determined gravimetrically. Followingly, $10 \mathrm{ml}$ of $4 \mathrm{M} \mathrm{HCl}$ was added and incubated at $60^{\circ} \mathrm{C}$ for 2 hours. The acid hydrolysed mass was stirred with $20 \mathrm{ml}$ of hexane: methanol mixture $(1: 1)$ at room temperature for 3 hours followed by centrifugation at $2,000 \mathrm{~g}$ for 5 minutes at room temperature to separate organic upper phase and aqueous lower phase [8]. All experiments were performed in triplicate. Followingly, upper phase containing lipids were recovered and evaporated using nitrogen gas and dry lipids were weighed. The yield of extracted material was determined and expressed as grams of extractable lipid per gram of dry solid.

\section{RESULTS AND DisCUSSION}

\section{A. Screening and Characterization of the Isolates for Lipid Accumulation}

The lipid accumulation capacity of the isolated colonies was revealed by Sudan Black B staining of the cultures grown in MGYP medium. The yeast strain were stained with Sudan Black B, the oleaginous cells show lipid bodies stained blue with the pink cytoplasmic background (lipids appearing as dark spots within lighter cytoplasm). Whereas, the non-oleaginous cells are stained completely pink (uniformly stained) as indicated in Fig.1.
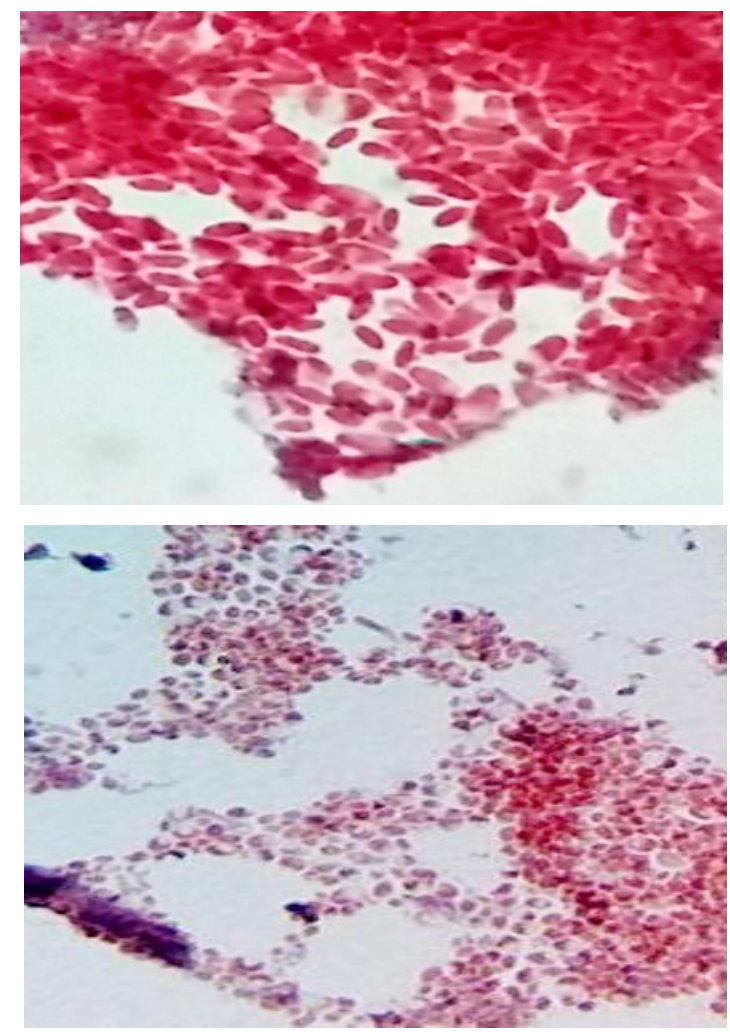

Figure 1. Cells stained by original Sudan Black B technique stained lipid matter surrounding the cytoplasmic membrane of non-oleaginous

(A) and oleaginous yeasts (B) observed with microscope.

The method of current isolation of oil fungi is Sudan Black B stain, which is cumbersome and time-consuming, and the result is greatly influenced by the staining. This method of isolating fat microorganism gives less false positive results, when compared with the results got form Sudan Black B dyeing separation. Moreover, it is faster and simpler [9], [10]. The oil accumulationg yeasts of the 10 strains is shown of Morphologcal characteristics in Table I.

\section{B. Oil Extraction}

The lipid-accumulating ability of the yeast strains is expressed as lipid content after 5 days of cultivation (Table II). The current research of oil production is mainly concentrated on the high concentration of glucose as carbon source. The best lipid concentration of strains CTWY19, CTWY24, CTWY02, CTWY07 and CTWY01 were 7.50, 6.06, 5.00, 4.90 and 4.54. The highest biomass strains were CTWY17, CTWY22, CTWY24, CTWY19 and CTWY01, which were 11.09, 10.93, 10.82, 10.47 and $8.82 \mathrm{~g} / \mathrm{L}$, respectively, Therefore, stain CTWY, CTWY and CTWY can utilize glucose as carbon source to produce oil well. In particular, CTWY showed the highest lipid content at 37.88-71.59\%. 
TABLE I. MORPHOLOGICAL AND LIPID PRODUCTION BY VARIOUS MiCROORGANISMS

\begin{tabular}{|c|c|c|c|c|c|c|}
\hline Strains & Sources & Colony color & Colony Margin & Colony Elevation & Colony Surface & Cell shape \\
\hline CTWY01 & Canteen Wastewater & Cream & Entire & Raised & Smooth & Oval \\
\hline CTWY02 & Canteen Wastewater & White & Undulate & Raised & Rough & Cylindrical-rod \\
\hline CTWY05 & Canteen Wastewater & White & Undulate & Umbonate & Smooth & Cylindrical \\
\hline CTWY06 & Canteen Wastewater & Cream & Entire & Raised & Smooth & Ellipsoidal \\
\hline CTWY07 & Canteen Wastewater & White & Undulate & Umbonate & Rough & Cylindrical \\
\hline CTWY17 & Canteen Wastewater & Salmon Red & Entire & Raised & Smooth & Oval \\
\hline CTWY19 & Canteen Wastewater & White & Entire & Convex & Smooth & Oval \\
\hline CTWY22 & Canteen Wastewater & Salmon Red & Entire & Pulvinate & Smooth & Oval \\
\hline CTWY23 & Canteen Wastewater & Salmon Red & Entire & Pulvinate & Smooth & Oval \\
\hline CTWY24 & Canteen Wastewater & Orange Red & Entire & Pulvinate & Smooth & Oval \\
\hline
\end{tabular}

TABLE II. The BIOMASS, LIPID AND LIPID CONTENT OF 10 OlEAGINOUS YeAST STRAINS

\begin{tabular}{|c|c|c|c|}
\hline Strains & Biomass $(\mathrm{g} / \mathrm{l})$ & Lipid concentration $(\mathrm{g} / \mathrm{l})$ & Lipid content $(\%)$ \\
\hline CTWY01 & 8.82 & 4.54 & 51.47 \\
\hline CTWY02 & 7.72 & 5.00 & 64.74 \\
\hline CTWY05 & 7.00 & 4.38 & 61.65 \\
\hline CTWY06 & 6.99 & 4.31 & 71.59 \\
\hline CTWY07 & 6.85 & 4.90 & 34.40 \\
\hline CTWY17 & 11.09 & 3.80 & 71.44 \\
\hline CTWY19 & 10.47 & 7.50 & 37.88 \\
\hline CTWY22 & 10.93 & 4.14 & 50.36 \\
\hline CTWY23 & 8.40 & 4.23 & 55.97 \\
\hline CTWY24 & 10.82 & 6.06 & \\
\hline
\end{tabular}

By applying Sudan Black B tests, 10 strains were identified as potential lipid biomass producer (Table I). The result showed that two oleaginous strains (CTWY07 and CTWY19) accumulated the highest lipid content were 71.59 and $71.44 \%$ of their dry biomass (morphology and cell shape in Fig. 2). This amount of lipid content ( 71.59 and $71.44 \%$ ) were greater than those taken different yeasts in other studies. For example, it had been reported that the oleaginous yeast $R$. toruloides $\mathrm{Y} 4$ in the flask culture reached a dry biomass of $151.5 \mathrm{~g} / \mathrm{l}$, however, the final cellular lipid content was only 48.0\%[11]. In addition, Yumauchi and colleagues [12] obtained a high cell density of $153 \mathrm{~g} / \mathrm{l}$ and a lipid content of $54 \%$ with $L$. starkeyi using a complicated feeding medium. When the culture medium lacks the nitrogen source, the isocitric dehydrogenase (ICDH) was suppresses, therefore the tricarboxylic acid circulation (TCA) was blocked. Extra carbon source was transformed to triglyceride (TAG) by a series of enzymes like the citric acid lytic enzyme, the malic acid enzyme, the fatty acid enzyme, thus completed the fat accumulation. In this article. Fat microorganisms were screen by the principle of high fat contents and fast growth on nitrogen limited culture medium [2].
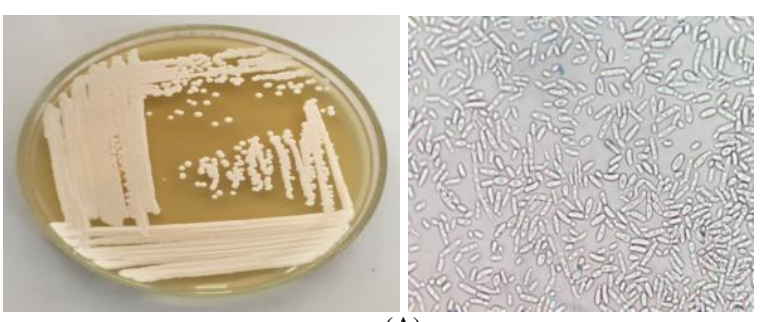

(A)

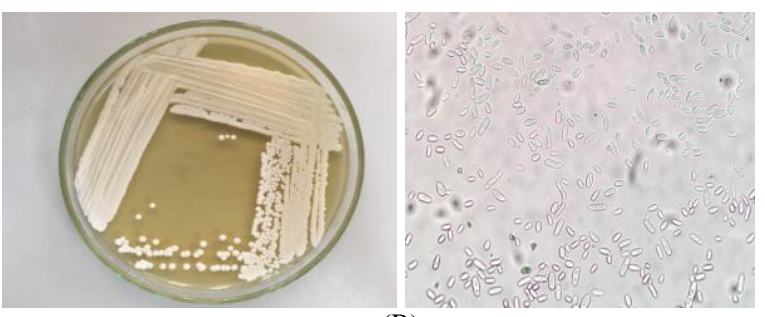

(B) 


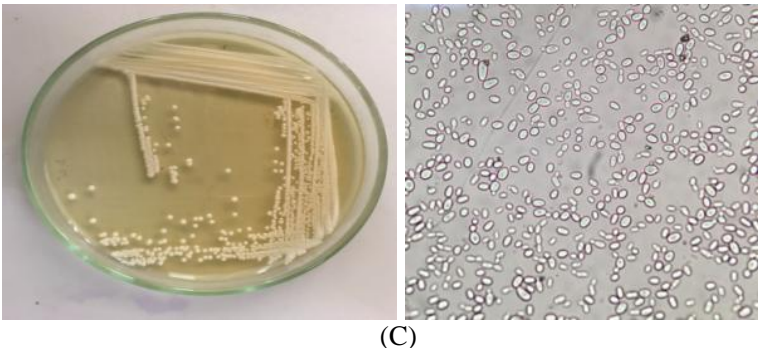

Figure 2. The colony morphology on YPD agar plate and cell shape under microscope 400X (A) CTWY02 (B) CTWY07 and (C) CTWY19.

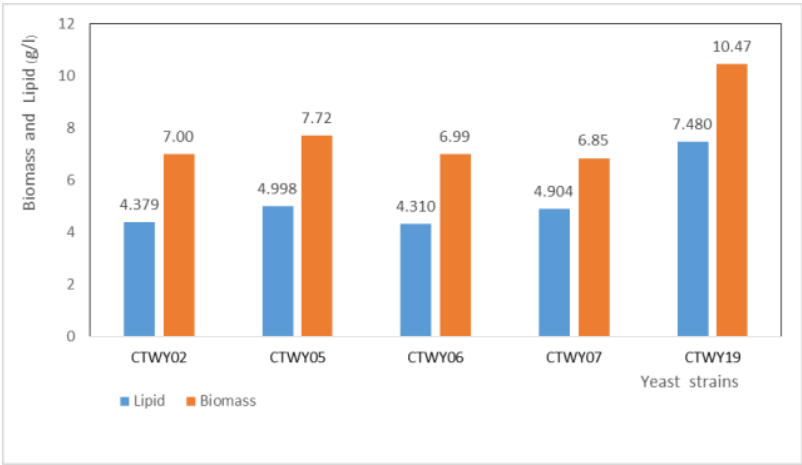

(A)

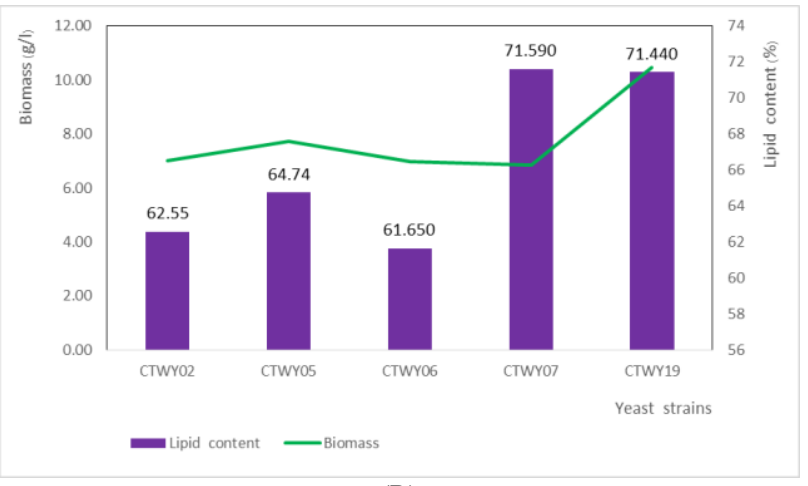

(B)

Figure 3. The biomass and Lipid (A) and Biomass and Lipid content (B) of the five oleaginous yeast strains cultivated in glucose by shaking flask cultivation at $150 \mathrm{rpm}$ and room temperature for $120 \mathrm{~h}$. The data represented averaged of three replicates.

Biomass, lipid concentration and lipid content are important indicators to evaluation of microbial oils. The result indicate that 10 strains the oil content of which was more than $20 \%$, and five strains that accumulated the high lipid levels of $61.65-71.59 \%$ of their dry biomasses (Figure 3).Lipid accumulation in oleaginous yeasts and fungi has been studied mainly with the aim to produce importance as potential source of triacylglyceride (TAG), poly-unsaturated fatty acids (PUFA) and as biodiesel precursors. In general, yeasts and molds can accumulate more lipids over bacteria and microalgae mainly due to rapid growth rate and therefore, have potential as commercial sources of oils. The accumulated lipids in yeasts get deposited as intracellular lipid bodies (LBs) [13].

\section{CONCLUSION}

Our results show that the efficiency of lipid recovery from extraction techniques. We found both species that already been reported as oleaginous yeasts. Evaluation of lipid production from glucose of the oleaginous yeast strains revealed that CTWY07 and CTWY19 were 71.59 and $71.44 \%$ of its dry biomass, respectively. These yeast species has the ability to utilize glucose and nitrogen compounds and to grow under stressful conditions. It was found that some of those impurities could even increase biomass concentration and lipid accumulation. Therefore, it is a promising oleaginous yeast candidate for production of biodiesel production. Acknowledgment

This work was supported by Department of Biology, Facultry of Science and Technology, Rajabhat MahaSarakham University.

\section{REFERENCES}

[1] P. Polburee, W. Yongmanitchai, N. Lertwattanasakul, T. Ohashi, K. Fujiyama, and S. Limtong, "Characterization of oleaginous yeasts accumulating high levels of lipid when cultivated in glycerol and their potential for lipid production from biodieselderived crude glycerol," Fungal Biology, vol. 119, pp. 1194-1204, 2015.

[2] S. L. Li, et al., "Isolation, identification and characterization of oleaginous fungi from the soil of Qinghai Plateau that utilize Dxylose," African Journal of Microbiology Research, vol. 5, no. 15, pp. 2075-2081, August 2011.

[3] F. Thevenieau and J. M. Nicaud, "Microorganisms as sources of oils," Oleagineux Corps Gras Lipides, vol. 20, no. 6, P. D603, 2013.

[4] G. Knothe, "A technical evaluation of biodiesel from vegetable oil vs.algae will algae-derived biodiesel perform,"Green Chem., vol.13, pp. 3048-3065, August 2011.

[5] A. Tanimura, et al., "Selection of oleaginous yeasts with high lipid productivity for practical biodiesel production," Bioresource Technology, vol. 153, pp. 230-235, 2014.

[6] S. Sankh, M. Thiru, S. Saran, and V. Rangaswamy, "Biodiesel production from a newly isolated Pichia kudriavzevii strain," Fuel, vol. 106, pp. 690-696, 2013.

[7] K. L. Burdon, J. C. Stokes, and C. E. Kimbrough, "Studies of the common aerobic spore-forming bacilli: I. staining for fat with Sudan Black B-sfranin,” J. Bacteriol., vol. 43, pp. 717-724, 1942.

[8] P. M. Nema and A. Kumari, "Isolation of lipid producing yeast and fungi from secondary sewage sludge and soil," Australian Journal of Basic and Applied Sciences, vol. 7, no. 9, pp. 283-288, 2013.

[9] L. X. Pan, D. F. Yang, L. Shao, W. Li, G. G. Chen, and Z. Q. Liang, "Isolation of the oleaginous yeasts from the soil and studies of their lipid-producing capacities," Food Techno. Biotechnol, vol. 47, no. 2, pp. 215-220, 2009.

[10] X. Chen, Z. H. Li, X. X. Zhang, F. X. Hu, D. Y. Dewey, and J. Bao, "Screening of oleaginous yeast strains tolerant to lignocellulose degradation compounds," Appl. Biochem. Biotechnol., vol. 159, pp. 591-604, 2009.

[11] Y. H. Li, Z. B. Zhao, and F. W. Bai, "High-density cultivation of oleaginous yeast Rhodosporidium toruloides Y4 in fed-batch culture," Enzyme Miicrob. Technol., vol. 41, no. 3, pp. 312-317, 2007.

[12] H. Yamauchi, H. Mori, T. Kobayashi, and S. Shimizu, "Mass production of lipids by Lipomyces starkeyi in microcomputeraided-fed-batch culture," Journal Ferment Technol., vol. 61, no. 3, pp. 275-280, 1983.

[13] A. Jape, A. Harsulkar, and V. R. Sapre, "Modified Sudan Black B staining method for rapid screening of oleaginous marine yeasts", International Journal of Current Microbiology and Applied Sciences, vol. 3, no. 9, pp. 41-46, 2014. 


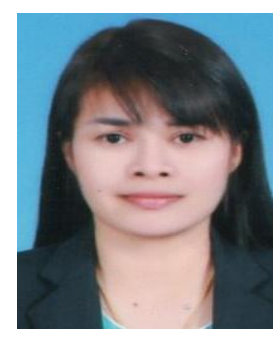

Kusumawadee Thancharoen was born in Chaiyaphoom Province, Thailand, 1980. She got her Master Degree of Microbiology, Kasetsart University, Bangkok, Thailand. She is Lecturer of Rajabhat MahaSarakham University, Thailand and her research interests are ethanol, biodiesel, metabolite of microbia.

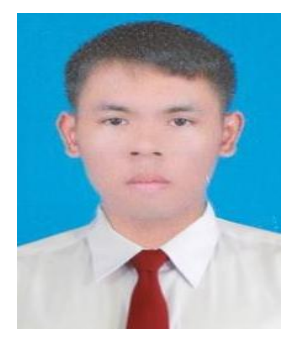

Auttaporn Malasri was born in Maha Sarakham Province, Thailand, 1994.

He got his Bachelor's Degree of Biology, Rajabhat MahaSarakham University,

MahaSarakham, Thailand. $\mathrm{He}$ is now a student of Rajabhat MahaSarakham University, Thailand. His research interests are screening oleaginous yeast and biodiesel.

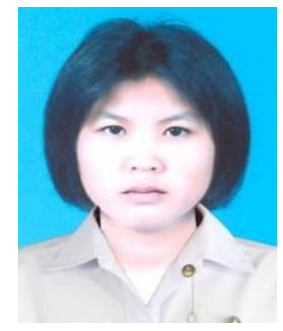

Walailak Leamsingkorn was born in Kalasin Province, Thailand, 1994. She got her Bachelor's Degree of Biology, Rajabhat MahaSarakham University, Maha Sarakham, Thailand. She is a Student of Rajabhat MahaSarakham University, Thailand. Her research interests are screening oleaginous yeast and biodiesel.

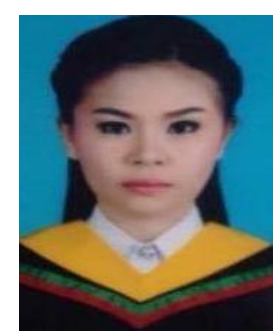

Patchareeporn Boonyalit was born in Ubonratchathanee Province, Thailand, 1994. She got her Bachelor's Degree of Biology, Rajabhat MahaSarakham University, Maha Sarakham, Thailand. She is a Teacher of Huaymarktai School, Thailand. Her research interests are screening of thermotolerant yeast and ethanol. 